\title{
Prolotherapy and Low Level Laser Therapy: A Synergistic Approach to Pain Management in Chronic Osteoarthritis
}

\author{
Vinicius Tieppo Francio, ${ }^{1,2,3,}$ Robert S Dima, ${ }^{4}$ Chris Towery, ${ }^{1}$ and Saeid Davani ${ }^{1}$ \\ ${ }^{1}$ University of Science, Arts and Technology - USAT College of Medicine, Olveston, Montserrat, BWI \\ ${ }^{2}$ Essential Integrative Health - Spine, Orthopaedics and Pain Management, Oklahoma City, OK, USA \\ ${ }^{3}$ Variety Care - Community Health Center, Oklahoma City, OK, USA \\ ${ }^{4}$ School of Interdisciplinary Sciences - McMaster University, Hamilton, ON, Canada \\ "Corresponding author: Vinicius Tieppo Francio, University of Science, Arts and Technology - USAT College of Medicine - Olveston, Montserrat, BWI. E-mail: \\ vinicius.francio@usat.edu
}

Received 2017 January 17; Revised 2017 June 02; Accepted 2017 October 09.

\begin{abstract}
Regenerative injection therapy and low level laser therapy are alternative remedies known for their success in the treatment and symptomatic management of chronic musculoskeletal conditions. In response to the growing demand for alternative therapies in the face of the opioid epidemic, the authors conduct a literature review to investigate the potential for prolotherapy and LLLT to be used adjunctively to manage chronic osteoarthritis (OA). OA is a degenerative chronic musculoskeletal condition on the rise in North America, and is frequently treated with opioid medications. The regenerative action of prolotherapy and pain-modulating effects of LLLT may make these two therapies well-suited to synergistically provide improved outcomes for osteoarthritis patients without the side effects associated with opioid use. A narrative descriptive review through multiple medical databases (Google Scholar, PubMed, and MedLine) is conducted, restricted by the use of medical subject headings. 71 articles were selected for reading in full, and 40 articles were selected for use in the study after reading in full. A review of the literature revealed good clinical results in the use of prolotherapy and LLLT separately to manage chronic musculoskeletal pain due to osteoarthritis and other chronic conditions. It is also recognized in the literature that prolotherapy works most effectively when used adjunctively with other treatments. Downsides to the use of prolotherapy include mild side effects of pain, stiffness and bruising and potential adverse events as a result of injection. This study is limited by the lack of clinical trials available involving both LLLT and prolotherapy injections used adjunctively, and by the low number of high impact literature concerning the treatment of (specifically) osteoarthritis by alternative methods. The authors suggest that practicing health care providers consider utilizing LLLT and prolotherapy together as a supplementary method in the management of chronic pain due to osteoarthritis, to minimize the long-term prescription of opioids and emphasize a less invasive treatment for this debilitating condition.
\end{abstract}

Keywords: Laser Therapy, Prolotherapy, Pain Management, Chronic Pain, Osteoarthritis

\section{Background}

Osteoarthritis is a degenerative, chronic musculoskeletal condition on the rise in North America, which is worrying since it presents a significant burden to public healthcare systems and is a major cause of chronic pain (1). Today, although alternative treatment methods exist, the most common way to manage chronic pain in osteoarthritis is to medicate with opioids (2). Since opioid medications are now recognized to have serious side effects, including sleep apnea, addiction and fatal overdose (3), it is now vital to further explore novel and effective ways to manage osteoarthritic pain without medical intervention.

The pathogenesis of osteoarthritis is well-understood to involve chondrocytic apoptosis, subchondral bone sclerosis, osteophyte development and persistent cytokines (4). A major etiology that initiates the development of osteoarthritic changes in a joint is ligamentous injury, which causes joint instability and biomechanical microstress (5). This key factor in the etiology of the condition justifies the use of prolotherapy to treat the osteoarthritic joint, by restoring functional stability through the proliferative strengthening of supporting ligamentous structures.

Prolotherapy (i.e. RIT, regenerative injection therapy) is an alternative treatment method wherein a type of "proliferative" solution (hyperosmolar dextrose, sodium morrhuate, and/or other compounds) is injected into and around the joint capsule, in order to stimulate the natural healing process of the intra-articular, capsular and adjacent ligamentous tissues (6). The proposed mechanisms of action for prolotherapy involve osmotic rupture of local cells, local cellular irritation and chemotactic attraction of inflammatory mediators (7). First implemented to treat hernias, today prolotherapy is a well-known method to manage and treat chronic musculoskeletal conditions like osteoarthritis, tendinosis and epicondylitis. Recent

Copyright @ 2017, Anesthesiology and Pain Medicine. This is an open-access article distributed under the terms of the Creative Commons Attribution-NonCommercial 4.0 International License (http://creativecommons.org/licenses/by-nc/4.0/) which permits copy and redistribute the material just in noncommercial usages, provided the original work is properly cited. 
advents to this technology include platelet-rich-plasmatherapy (PRP), stem cell injection (8) and prolozone (ozone + prolotherapy) (9).

Another well-known intervention used for pain management is low-level laser therapy (LLLT), or cold laser therapy, which is a form of photo-biomodulation therapy in which low-frequency continuous laser of typically 600 $1000 \mathrm{~nm}$ wavelength (10) is used for pain reduction and healing stimulation. Recent literature has explored LLLT efficacy in treating musculoskeletal injury, post-surgical wound healing and chronic inflammatory joint conditions. Also, LLLT has long since been observed to improve cell proliferation and collagen synthesis in laboratory studies. In particular, fibroblastic proliferation is a hallmark feature of low level laser exposure (11). LLLT is known to act on photoreceptors on the mitochondria of cells, with observed effects including enhanced production of adenosine triphosphate (ATP), increased oxygen metabolism, increased concentration of reactive oxygen species and increased concentration of nitric oxide (NO) $(12,13)$.

\section{Objectives}

In response to the need for further research into alternative treatment methods for chronic osteoarthritis given the current opioid epidemic, this paper seeks to provide, for practicing health care providers, a review of the literature regarding the current use of prolotherapy and LLLT in treating chronic musculoskeletal conditions. In this review, the authors seek to draw connections between prolotherapy and LLLT mechanisms, in order to gauge their potential to act synergistically in the management of the osteoarthritic joint.

\section{Methods}

The present study is a descriptive study specific on the utilization of low-level laser therapy (LLLT) and hyper dextrose prolotherapy injection therapy and its clinical applications in chronic pain due to osteoarthritis. The study was designed to answer the specific question postulated by the authors that, for the symptomatic management of chronic pain due to OA, the utilization of hyper dextrose prolotherapy injections and LLLT may be a reasonable intervention based on recent results presented in the modern literature. The authors intentionally do not present a results section stand alone, but combine the results of this descriptive narrative review with the discussion section. The study describes the data results and characteristics about the phenomenon (chronic pain and osteoarthritis) and the clinical applications (use of prolotherapy and low level laser therapy) being studied utilizing a clinician's perspective and not a meta-analytical or statistical review of the literature. Therefore, we present a discussion of the results summarized by this descriptive narrative review in which the main key point is to help clinicians that currently must decide between standard invasive therapies with steroids, chronic opioid therapy, surgery, versus new cutting edge regenerative options, such as prolotherapy and supporting alternative and complementary therapies, such as laser therapy, discussing the pros and cons of such decision-making process.

The present study was conducted as a narrative descriptive review of the literature utilizing the following search engines: Medline, PubMed, Google Scholar, Index Copernicus, EBSCO, and Science Direct. Only peer-reviewed articles were used in this review. We did not utilize the EMBASE database due to cost and limited access, although cross-references between different databases provided a wide access to resources. The search was restricted to publications from 1980 to 2016. The search was limited to studies published in the English language with the Medical subject headings (MeSH) "osteoarthritis"; "pain"; "sclerotherapy"; "lasers". If other studies were found with the MeSH heading but were outside the scope of this paper, they were included in the deliberation process, but excluded for references and not utilized in this manuscript.

The search strategy for this review was as follows: step 1: the reference lists of articles identified by the search engines were searched and triaged into usable relevant publications. Step 2: the relevant research articles were then reviewed by the author(s) and summarized into a relevant table of research. Peer reviewed articles were included or excluded based on scope, language, relevancy and content pertinent to the publication. Step 3: the author(s) reviewed the articles and summarized the information into the present narrative review, emphasizing a clinician's standpoint regarding the pros and cons of symptomatic management of chronic pain due to osteoarthritis regenerative medicine options, such as prolotherapy and complementary low-level laser therapy.

\section{Discussion}

\subsection{Osteoarthritis- Overview}

Although ascertaining the incidence and epidemiology of osteoarthritis (OA) is challenging, recent literature suggests that a substantial proportion of adults worldwide are affected, and the condition has been on the rise within the last decade. It is estimated that the prevalence of knee joint osteoarthritis for example is approximately $10 \%$ in men and $13 \%$ in women (14). Osteoarthritis is known to 


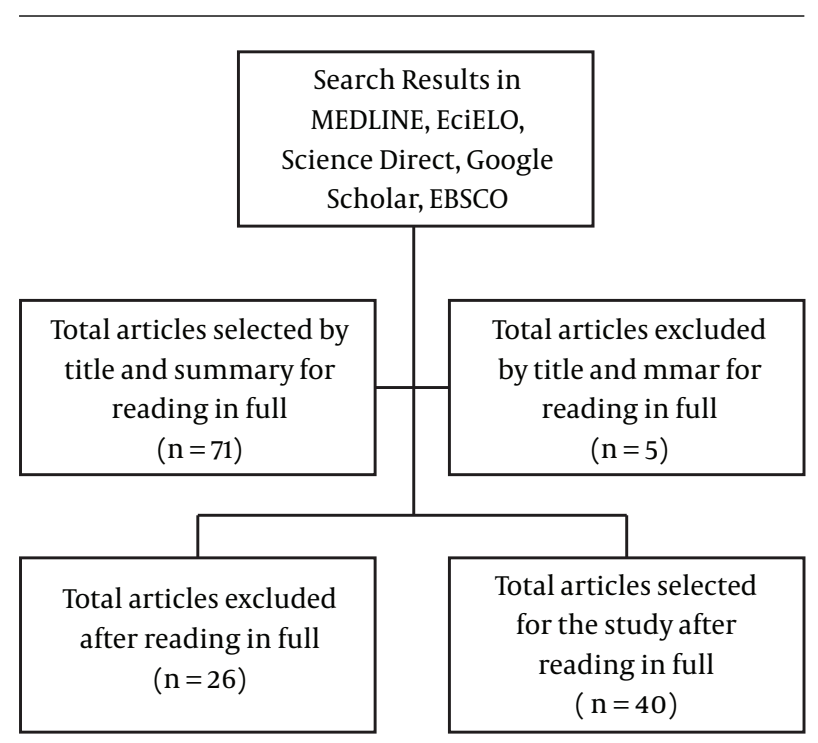

Figure 1. Flow Diagram of Review System and Selection of Sources

be one of the major types of musculoskeletal conditions to contribute significantly to chronic disability (15). Studies have linked chronic osteoarthritis with chronic pain, sleep disturbance and depressive symptoms, further accentuating the importance of proper management of this very common condition (16). Weight-bearing joints are frequently affected by osteoarthritis, and include the hip, knee, shoulders, and the spine. This condition has increased in prevalence over the last quarter century and is expected to continue rising, further burdening the healthcare system and decreasing patient's quality of life (17).

To understand the rationale behind treatment methods for OA, it is vital to have an understanding of the pathophysiology of the condition. Osteoarthritis is characterized by a loss of the functional/biochemical integrity of a joint (18). Perhaps the most iconic feature of osteoarthritis, and a key pathological feature, is the degeneration of articular cartilage within the joint. Decreased articular cartilage, subchondral bone sclerosis, osteophyte development and chronic low-grade synovial inflammation resulting from a history of repetitive ligamentous microtrauma are all involved in the pathogenesis of $\mathrm{OA}$, and result in the main symptoms of OA; joint stiffness, joint pain and joint dysfunction (4). Joint dysfunction and pain are the main clinical symptoms of $\mathrm{OA}$, and are thought to be due to nervous aggravation of "A" delta mechanoreceptors in the fibrous capsule of joints, and "C" polymodal nerve endings in the synovium and surrounding joint components (18). Contributing factors to the development of osteoarthritis involve increased age, obesity (estimated to increase risk of OA 3-fold (14)) injury, occupation, developmental deformi- ties and joint laxity.

\subsection{Osteoarthritis- Pathophysiology}

It is important to note that joint laxity has been found to be a major contributor to the development of osteoarthritis. Joint laxity compromises the otherwise natural balance and stability of the joint, leading to unhealthy weight-bearing changes within the joint and the development of osteoarthritic changes (19). The biochemical properties of osteoarthritis relate to an abnormal remodelling of the joint, spurred by inflammatory mediators. Cytokines such as IL-1, TNF, IL-8, bioactive lipids like PGE2, as well as other well-known pro-inflammatory chemicals like nitric oxide and vasoactive-intestinal peptide(VIP) are seen in high concentration in the osteoarthritic joint; these elevated pro-inflammatory markers are likely attributed to the synovitis associated with osteoarthritis, and contribute to the holistic degeneration of the joint (4). The chronic phase of osteoarthritis is characterized by decreased chondrocyte proliferation, chondrocytic apoptosis, osteophyte formation and bone sclerosis.

Treatment of osteoarthritis is currently, and has long been, aimed at relieving the symptoms of the condition. Non-pharmacological management, medical intervention and surgical intervention are generally pursued, in that order, in the treatment of OA (4). Conservative management generally includes physical therapy, acupuncture, injection therapy, NSAIDs, and opioid therapy. On the other hand, aggressive options for the symptomatic management of OA include nerve blocks, radiofrequency neural ablation, arthroscopic lavage, osteotomy and joint replacement.

Unfortunately, in patients with chronic osteoarthritis, treatment options are often relegated to chronic pain management with opioids, which are known to create detrimental side effects and even cause addiction and death in chronic pain management patients $(20,21)$. Front page headlines in North America describe the opioid crisis as "pervasive in every population, urban and rural, young and old, rich and poor". In particular, the United States and Canada are known to have the highest per capita volume of opioids like hydromorphone, codeine and fentanyl dispensed in the world. Today, current opioid overdose rates are staggering; with 2 people dying every day from overdoses alone. In 2014, 1400 died in the US by opioid overdose (22). Other journalistic reports suggest an immediate need to address chronic pain and opioid utilization appropriately, with a comprehensive treatment approach to manage chronic pain and, particularly, the need to consider supplementary and alternative therapies to pharmaceutical intervention (23). 
Other treatment options for osteoarthritis comprise their own subset of treatment type. These supplementary treatment options for osteoarthritis include regenerative injection therapy (RIT), low level laser therapy (LLLT), hyaluronic acid injections, hydrotherapy, physical therapy, prolozone therapy and others. In this article, we focus on RIT (specifically prolotherapy) and LLLT (or cold laser therapy) to summarize their observed effects on chronic osteoarthritis.

The pathophysiology of osteoarthritis, initiated by ligamentous trauma, is illustrated in Figure 2. Hallmark osteoarthritic changes are emphasized, like articular cartilage degeneration occurring due to the action of intraarticular proteases and compensatory osteophyte development.

\subsection{Regenerative Injection Therapy- Overview}

Regenerative injection therapy (RIT), or prolotherapy, is a supplementary regenerative treatment of, among others, chronic musculoskeletal conditions. Prolotherapy injection utilizes a hypertonic dextrose solution (among other substances) injected intra and/or extra-articularly to initiate a brief inflammatory cascade stimulating native healing and subsequent tissue growth; with restoration of tissue integrity, including organized connective tissue in previously injured ligaments, thickening of collagen bundles, and increase in energy absorption within the joint (24).

\subsection{Regenerative Injection Therapy-Mechanisms}

Specifically, prolotherapy is known to target and resolve ligament laxity, a major contributor to osteoarthritis development, by initiating an inflammatory cascade to attract fibroblasts that encourage collagen synthesis, which reinforces connective tissue at the affected area (19). It is theorized that the attraction of inflammatory mediators and the release of growth factors could be due to the action of the hyperosmolar dextrose solution acting as a cellular irritant (6). The resulting strengthening of the ligaments supporting the joint is thought to return functional biomechanics to the joint and result in pain relief. Prolotherapy injections have also recently been seen to have disease-modifying effects in knee OA, specifically by stimulating the metabolic activation of cartilage, resulting in the growth of fibro-like and hyaline-like cartilage (24). These new findings are especially encouraging to the role of prolotherapy in treating osteoarthritis, of which the classic appearance is insufficient articular cartilage, and gives credence to recent findings in prolotherapy suggesting a direct anabolic effect on the proliferation of chondrocytes (25). Recent studies validate the continued use of this treatment method for knee osteoarthritis (26) Achilles tendinosis (27), lateral epicondylitis (28) and other chronic musculoskeletal injuries (19). Furthermore, a direct pain-modulating effect has also been associated with prolotherapy injections, suggesting that hypertonic dextrose may decrease pain via a sensorineural mechanism through direct exposure of dextrose and mannitol to multiple intra-articular pain generators, including the fat pad, synovium, and menisci (24).

Although various cocktails of prolotherapy are theoretically possible in the use of regenerative therapy, the most common solutions used in the treatment of chronic musculoskeletal pain include $12 \%$ - $15 \%$ up to $25 \%$ hyperosmolar dextrose (glucose), mannitol and Traumeel $(19,28)$. Also, recent literature has supported the use of certain variations of prolotherapy; platelet-rich plasma, stem cell therapy, and prolozone (prolotherapy plus ozone gas) which are all modified forms of regenerative injection therapy (RIT) and have proved their usefulness in clinical trials (8, 9). The utilization of Traumee ${ }^{\circledR}$ solution (a natural homeopathic anti-inflammatory) with intra-articular prolotherapy has demonstrated in vitro modulatory effects on human T-cells and monocytes and their ability to secrete proinflammatory mediators, such as interleukin-1beta (IL-1 $\beta$ ), tumor necrosis factor alpha (TNF $\alpha$ ), and interleukin-8 (IL8). Traumeel was found to modulate the secretion of these mediators, inhibiting secretion in resting and activated cells by up to $54 \%$ - 70\%. Furthermore, Traumeel appears to act by regulating the orchestration of the overall process of acute local inflammation, speeding up the healing process, regulating effects on lymphocyte synthesis of the inhibitory cytokine TGF- $\beta$ synthesis and other pro- inflammatory T-lymphocytes (for example, TNF $\alpha$ and IL-1) (29).

The commonality between these various forms of prolotherapy is that a proliferative solution is injected into the joint space, inducing the natural repair of connective tissue in order to restore the integrity of a compromised joint. These RIT methods Induce the release of high concentrations of a variety of growth factors and cytokines important for angiogenesis, cell replication and myoblast/fibroblast proliferation. Noteworthy factors in this process include PDGF (platelet-derived growth factor), TGF B-1 (transforming growth factor beta-1), VEGF (vascular endothelial growth factor) and ILGF-1 (insulin-like growth factor-1), specially for PRP (8). Stem cell therapy denotes the use of adult mesenchymal (MSCs) or bone marrow aspirate iliac crest stem cells (BMAICSCs) to promote healing in terms of cartilage repair, angiogenesis, muscle and nervous regeneration. Additionally, the use of stem cells has been made more available through the advent of retrieving adult stem cells from adipose tissue, bone marrow and other sources. 

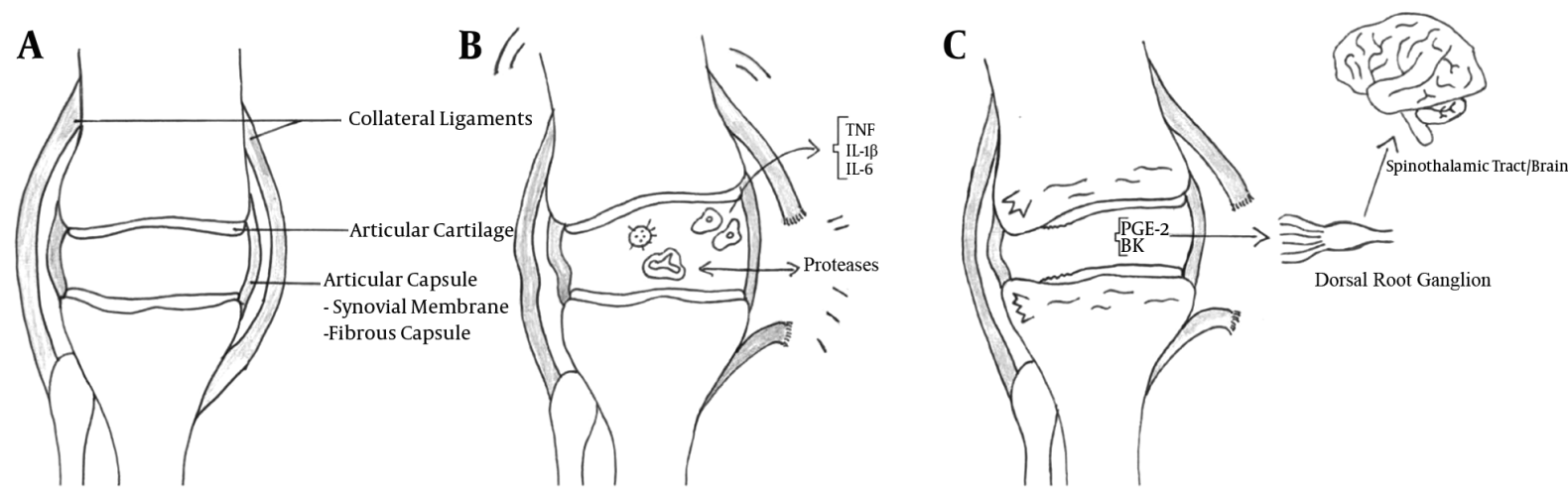

A, Simplified schematic representation of normal anatomy of the knee joint, with some of the major anatomical structure labeled; B, Ligamentous damage stimulates macrophages from the synovium to release inflammatory cytokines. The migration of T-cells and neutrophils from the surrounding vasculature causes an increased concentration of proteases intra-articularly; C, The osteoarthritic joint demonstrates deficient articular cartilage, subchondral sclerosis, osteophyte formation as well as decreased joint space. Pain signalling molecules are persistent in the affected joint, and stimulate sensorineural fibers in the fibrous capsule (A-delta mechanoreceptors) and in the synovium (C polymodal nerve endings), causing chronic pain.

\subsection{Regenerative Injection Therapy-Results}

Hyper-dextrose prolotherapy in its various forms presents a holistic approach to the treatment of the osteoarthritic joint by correcting ligamentous dysfunction, the root of the pathogenesis of osteoarthritis, without the risk of serious side effects or addiction inherent to opioid pain management (30). The current literature considers prolotherapy a viable option in the treatment of painful musculoskeletal conditions, particularly when other standard treatments have proved ineffective. A 2007 Cochrane review of prolotherapy effectiveness in the presence of chronic low back pain stressed that prolotherapy appears to be most effective at reducing pain in the presence of other adjunctive therapies, such as exercise and glucosamine and chondroitin oral supplementation (31).

Exercise and musculoskeletal manipulation are common therapies used adjunctively with prolotherapy (especially in the presence of nonspecific low back pain). Exercise therapy has been seen to be as effective as to other conservative treatments for lower back pain, hip and knee OA (32). However, these therapeutic options do have their disadvantages, since it is highly dependent on patient compliance and willingness to participate (33). At-home exercise therapy effectiveness is patient-dependent and subject to variation in implementation. Thus, it is important to explore the feasibility of other, less compliance-reliant therapies as adjunctive therapies to prolotherapy injections.

According to a post poll involving 171 practitioners, side effects of prolotherapy administration for back and neck pain included pain at administration, stiffness and bruis- ing. Adverse effects requiring hospitalization were overwhelmingly attributed to needle injuries, either causing spinal cord insult, temporary systemic reactions, hemorrhage or pneumothorax (30).

\subsection{LLLT- Overview}

One supplementary interventional therapy that has shown promise in clinical and laboratory studies is low level laser therapy (LLLT) or cold laser therapy. Laser biostimulation has been used for years in wound healing and has been most frequently used for post-surgical healing, skin wound healing and pain (34). The applications of cold laser therapy in deeper wound healing has been of interest in the scientific community, with literature exploring its potential in Achilles tendinopathy (27), myofascial neck pain (35) and recently, Bouchard's and Heberden's Osteoarthritis (36). In cold laser therapy, non-coherent light of wavelength $600-1000 \mathrm{~nm}$ is applied to an area of injury, in which photostimulation of the tissues promotes and accelerates healing and cellular function. The mechanism of LLLT has been studied at length in laboratory studies and clinical trials, and most emphasized in the literature is the mechanism of the cytochrome c oxidase (CCO) protein in the electron transfer chain (ETC) of mammalian cells as the main photo acceptor molecule that allows for photobiomodulation and its biological effect. This is evidenced by the similarity in absorption spectra between oxidized cytochrome c oxidase and action spectra from biological responses to light (37). 


\subsection{LLLT-Mechanisms}

Light from the cold laser is thought to interact with the copper and iron chromophores of cytochrome c to increase oxidation velocity and consequently accelerate cellular metabolism and the production of ATP. Increased levels of NO due to photostimulated dissociation of NO from CCO (normally facilitated by mitochondrial NO synthase) prevents the downregulation of oxygen metabolism; by this method, light-induced dissociation both increases the rate of ATP production and allows more free nitric oxide to be available as a vasodilator $(12,13)$. Furthermore, increased ATP production is known to cause increased concentrations of reactive oxygen species (ROS's), and these ROS's activate transcription factors leading to the upregulation of genes related to cellular proliferation and the production of cytokines and growth factors $(12,13)$. LLLT has also been seen to reduce the concentration of pain markers in a dose dependent manner, lowering the levels of biochemical markers like PGE2, IL-1B and TNF alpha (38). These theories can help to explain the findings of increased levels of ATP synthesis, oxygen uptake and RNA and protein synthesis seen in individual mitochondria, and the effect of fibroblastic proliferation, pain reduction and healing observed on a more macroscopic level $(11,13$, 39). Vasodilation, upregulation of genes related to cellular proliferation, and increased availability of oxygen, antiinflammatory cytokines and growth factors all contribute to the improved healing and strengthening of compromised ligaments and soft tissues.

\subsection{LLLT- Results}

In clinical studies, LLLT has shown promise in managing Achilles tendonitis (40), neck pain (35) and other chronic musculoskeletal chronic pain disorders (10). A recent 2016 study by Baltzer, Ostapczuk and Stosch gives compelling support for LLLT in the treatment of extremity osteoarthritis; reporting that LLLT exposure to Heberden's and Bouchard's OA had very large effects to significantly reduce pain and swelling at the treatment site (36).

Barriers to the implementation of LLLT in treating chronic pain in osteoarthritis involve the high variation in methods of application. From types of lasers to the specific wavelength used, health care providers may find the diverse options available to them intimidating. As per our review, there does not appear to be any distinct wavelength at which healing is optimal, as long as practitioners adhere to the flexible range of $600-1000 \mathrm{~nm}$.

\subsection{Conclusions}

The high and increasing incidence of chronic pain due to osteoarthritis is one of the leading causes of disability in North America. Current common treatment methods for this potentially debilitating condition often involve the prescription of opioid medications, which have been seen in association with significant side effects and the risk of medication addiction, which is already of an epidemic concern in the United States and Canada. Consequently, it is important that health care providers are aware of and consider supplementary treatment methods that are effective in treating chronic pain due to OA, without debilitating side effects like those of opioids.

Prolotherapy injections have demonstrated a solid chondrogenic effect in joint regeneration and a direct pain-modulating effect, suggesting that hypertonic dextrose may decrease pain via a sensorineural mechanism through direct exposure of dextrose and mannitol (among other proliferative solutions) to multiple intra-articular pain generators. In randomized clinical trials, prolotherapy has been shown to improve joint mobility, quality of life and reported musculoskeletal function. Furthermore, both LLLT and prolotherapy have been seen to significantly reduce joint pain and stimulate fibroblastic regeneration in clinical trials and laboratory experiments, superior to placebo and other standard conservative treatments, including improved reported patient outcomes in musculoskeletal function and fewer side effects. Given the potentially synergistic nature of the proliferative effects of prolotherapy and pain-modulating and healing effects of LLLT, the authors encourage further investigation into the cooperative use of these two well-known therapies in the treatment of chronic osteoarthritis. We feel that this may offer a partial solution to minimize the longterm prescription of opioids and emphasize a less invasive treatment for chronic osteoarthritis. The key point of this narrative review is to help clinicians that must decide between chronic opioid therapy for OA with significant side effects; standard injection and surgical interventions; and the newly cutting edge laser therapy and regenerative medicine alternatives for management of chronic pain due to osteoarthritis, and the pros and cons of such decision-making process, including its impact within the pain physician's practice.

\subsection{Limitations}

This study has potential limitations. We present a narrative review with discussion of the results with a clinical perspective and not a statistical standpoint. There are already currently published studies presenting actual data comparing the interventions we described here in innumerous conditions, as well as reviews of composite data with this scope. Therefore, we present in this study a clinical perspective of the utilization of prolotherapy and regenerative medicine options for the management of pain 


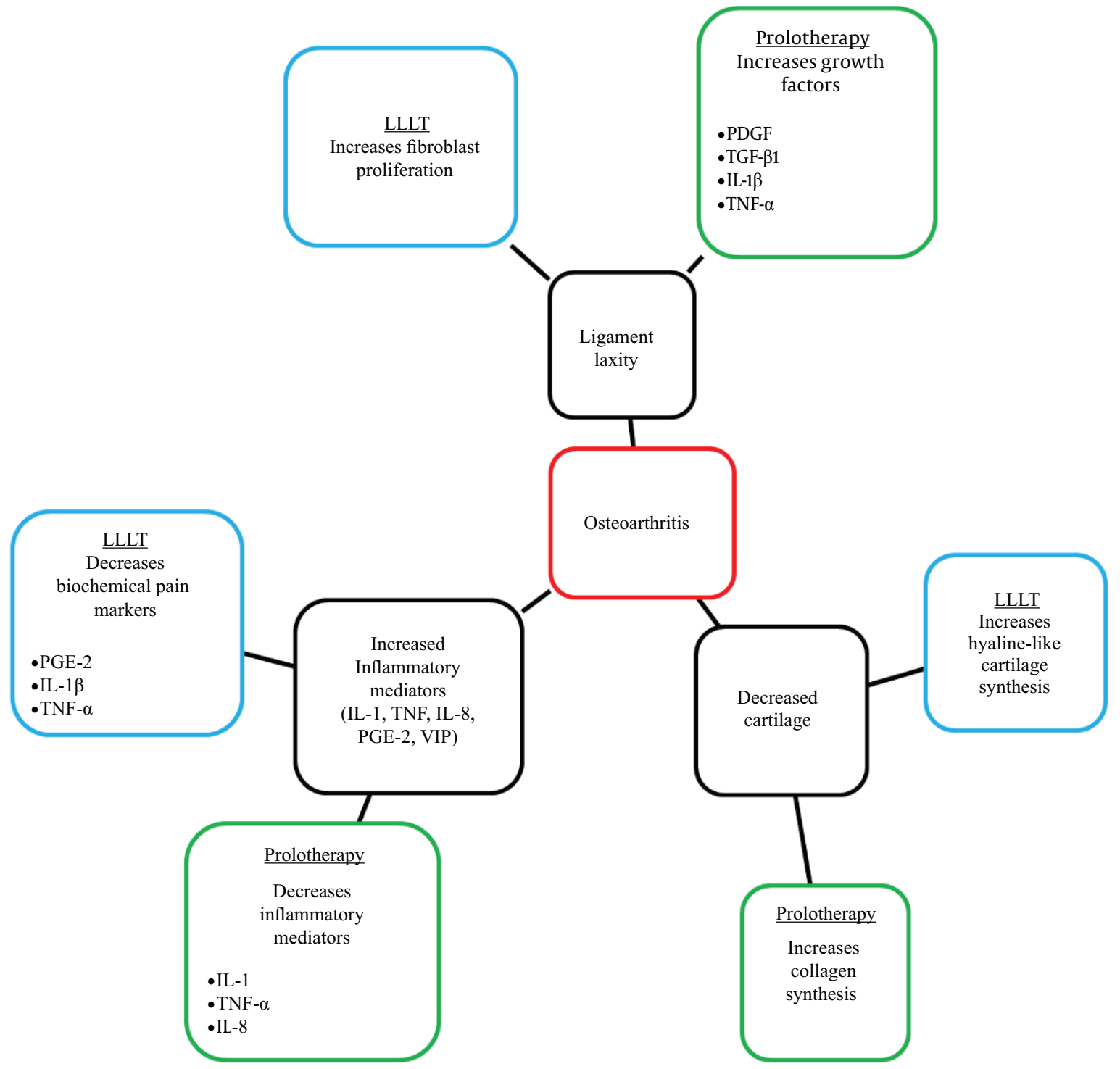

The above map offers a representation of the 3 main pillars of osteoarthritis pathophysiology; ligamentous laxity, articular cartilage degradation and the excessive presence of inflammatory mediators (IL-1, TNF, IL-8, PGE-2, VIP) and the mechanism by which prolotherapy and low level laser therapy likely contribute to the physiological benefits of osteoarthritis symptom management and joint support.

due to osteoarthritis, rather than a meta-analysis or stateof-the-art review of the literature of statistical and epidemiological results that would be mirroring other published studies. Hence our goal was to focus on clinical aspects by reviewing published data with focal emphasis to medical providers and the decision making process in managing OA pain. The key point of this narrative review is to help clinicians that must decide between chronic opioid therapy for OA with significant side effects; standard injection and surgical interventions; and the newly cutting edge laser therapy and regenerative medicine alternatives for management of chronic pain due to osteoarthritis, and the pros and cons of such decision-making process, including it's impact within the pain physician's practice. 


\section{Footnote}

Implication: Health care practitioners may benefit from this review by learning more about alternative therapies for chronic osteoarthritis to avoid the use of opioid medications. Increasing awareness about the scientifically supported advantages to alternative treatments reduce stigma surrounding these treatments and may improve patient outcomes by offering a partial solution to the current opioid epidemic.

\section{References}

1. Lawrence RC, Felson DT, Helmick CG, Arnold LM, Choi H, Deyo RA, et al. Estimates of the prevalence of arthritis and other rheumatic conditions in the United States. Part II. Arthritis Rheum. 2008;58(1):26-35. doi: 10.1002/art.23176. [PubMed: 18163497].

2. Boudreau D, Von Korff M, Rutter CM, Saunders K, Ray GT, Sullivan MD, et al. Trends in long-term opioid therapy for chronic noncancer pain. Pharmacoepidemiol Drug Saf. 2009;18(12):1166-75. doi: 10.1002/pds.1833. [PubMed: 19718704].

3. Walker JM, Farney RJ, Rhondeau SM, Boyle KM, Valentine K, Cloward TV, et al. Chronic opioid use is a risk factor for the development of central sleep apnea and ataxic breathing. J Clin Sleep Med. 2007;3(5):45561. [PubMed: 17803007].

4. Arden N, Blanco F, Cooper C, Guermazi A, Hayashi D, Hunter D, et al. Atlas of Osteoarthritis. Tarporley: Springer; 2014.

5. Hauser RA, Hauser MA, Baird NM, Martin DJ. Prolotherapy: an alternative to knee surgery. Journal of Prolotherapy. 2010;2(1):272-81.

6. Distel LM, Best TM. Prolotherapy: a clinical review of its role in treating chronic musculoskeletal pain. PM R. 2011;3(6 Suppl 1):S78-81. doi: 10.1016/j.pmrj.2011.04.003. [PubMed: 21703585].

7. Rabago D, Slattengren A, Zgierska A. Prolotherapy in primary care practice. Prim Care. 2010;37(1):65-80. doi: 10.1016/j.pop.2009.09.013. [PubMed: 20188998].

8. Alderman DD, Alexander R. Advances in regenerative medicine: highdensity platelet-rich plasma and stem cell prolotherapy for musculoskeletal pain. Pract Pain Manag. 2011;11(8).

9. Shallenberger F. ProlozoneTM-regenerating joints and eliminating pain. J Prolo. 2011;3(2):630-8.

10. Bjordal JM, Couppe C, Chow RT, Tuner J, Ljunggren EA. A systematic review of low level laser therapy with location-specific doses for pain from chronic joint disorders. Aust J Physiother. 2003;49(2):107-16. doi: 10.1016/S0004-9514(14)60127-6. [PubMed: 12775206].

11. Medrado AR, Pugliese LS, Reis SR, Andrade ZA. Influence of low level laser therapy on wound healing and its biological action upon myofibroblasts. Lasers Surg Med. 2003;32(3):239-44. doi: 10.1002/lsm.10126. [PubMed:12605432].

12. Chung H, Dai T, Sharma SK, Huang YY, Carroll JD, Hamblin MR. The nuts and bolts of low-level laser (light) therapy. Ann Biomed Eng. 2012;40(2):516-33. doi: 10.1007/s10439-011-0454-7. [PubMed: 22045511].

13. Hamblin MR, Demidova TN. Mechanisms of low level light therapy Proc. San Jose, CA: SPIE; 2006

14. Allen KD, Golightly YM. State of the evidence. Curr Opin Rheumatol. 2015;27(3):276-83. doi: 10.1097/BOR.0000000000000161. [PubMed: 25775186].

15. Neogi T, Zhang Y. Epidemiology of osteoarthritis. Rheum Dis Clin North Am. 2013;39(1):1-19. doi:10.1016/j.rdc.2012.10.004. [PubMed: 23312408].

16. Parmelee PA, Tighe CA, Dautovich ND. Sleep disturbance in osteoarthritis: linkages with pain, disability, and depressive symptoms. Arthritis Care Res (Hoboken). 2015;67(3):358-65. doi: 10.1002/acr.22459. [PubMed: 25283955].
17. Wheaton $\mathrm{M}$, Jensen $\mathrm{N}$. The ligament injury connection to osteoarthritis (extended version). J Prolo. 2011;3(4)

18. Kean WF, Kean R, Buchanan WW. Osteoarthritis: symptoms, signs and source of pain. Inflammopharmacology. 2004;12(1):3-31. doi: 10.1163/156856004773121347. [PubMed: 15035776].

19. Alderman D. Prolotherapy for knee pain. Pract Pain Manag. 2007;7(6):70-9.

20. Fishman SM. Opioid side effects, addiction, and anti-inflammatory medications. J Pain Palliat Care Pharmacother. 2005;19(1):51-5. doi: 10.1080/J354v19n01_09.[PubMed: 15814515].

21. Fishbain DA, Cole B, Lewis J, Rosomoff HL, Rosomoff RS. What percentage of chronic nonmalignant pain patients exposed to chronic opioid analgesic therapy develop abuse/addiction and/or aberrant drug-related behaviors? A structured evidence-based review. Pain Med. 2008;9(4):444-59. doi: 10.1111/j.1526-4637.2007.00370.x. [PubMed: 18489635].

22. Gomes T. Opioid Crisis should be on top of federal health agenda. Hospital News 2016. Available from: https://issuu.com/hospitalnews/ docs/flip_hospitalnews_oct2016.

23. Wallace G. Tackling the misuse of opioids; safe prescribing. Hospital News 2016. Available from: https://issuu.com/hospitalnews/docs/ flip_hospitalnews_oct 2016 .

24. Topol GA, Podesta LA, Reeves KD, Giraldo MM, Johnson LL, Grasso R, et al. Chondrogenic Effect of Intra-articular Hypertonic-Dextrose (Prolotherapy) in Severe Knee Osteoarthritis. PMR. 2016;8(11):1072-82. doi: 10.1016/j.pmrj.2016.03.008. [PubMed: 27058744].

25. Bertram KL, Krawetz RJ. Osmolarity regulates chondrogenic differentiation potential of synovial fluid derived mesenchymal progenitor cells. Biochem Biophys Res Commun. 2012;422(3):455-61. doi: 10.1016/j.bbrc.2012.05.015. [PubMed: 22579684].

26. Rabago D, Patterson JJ. Prolotherapy: an effective adjunctive therapy for knee osteoarthritis. J Am Osteopath Assoc. 2013;113(2):122-3. [PubMed: 23412672].

27. Tumilty SJ. Low level laser therapy for the treatment of tendinopathy with emphasis on the achilles tendon. University of Otago; 2010.

28. Scarpone M, Rabago DP, Zgierska A, Arbogast G, Snell E. The efficacy of prolotherapy for lateral epicondylosis: a pilot study. Clin J Sport Med. 2008;18(3):248-54. doi: 10.1097/JSM.0b013e318170fc87. [PubMed: 18469566].

29. Schneider C. Traumeel - an emerging option to nonsteroidal antiinflammatory drugs in the management of acute musculoskeletal injuries. Int J Gen Med. 2011;4:225-34. doi:10.2147/IJGM.S16709. [PubMed: 21556350].

30. Dagenais S, Ogunseitan O, Haldeman S, Wooley JR, Newcomb RL. Side effects and adverse events related to intraligamentous injection of sclerosing solutions (prolotherapy) for back and neck pain: A survey of practitioners. Arch Phys Med Rehabil. 2006;87(7):909-13. doi: 10.1016/j.apmr.2006.03.017. [PubMed:16813776].

31. Dagenais S, Yelland MJ, Del Mar C, Schoene ML. Prolotherapy in jections for chronic low-back pain. Cochrane Database Syst Rev 2007(2):CD004059. doi: 10.1002/14651858.CD004059.pub3. [PubMed: 17443537].

32. Hayden JA, van Tulder MW, Malmivaara AV, Koes BW. Meta-analysis: exercise therapy for nonspecific low back pain. Ann Intern Med. 2005;142(9):765-75. [PubMed: 15867409].

33. Schneiders AG, Zusman M, Singer KP. Exercise therapy compliance in acute low back pain patients. Man Ther. 1998;3(3):147-52. doi: 10.1016/s1356-689x(98)80005-2.

34. Conlan MJ, Rapley JW, Cobb CM. Biostimulation of wound healing by low-energy laser irradiation. A review. J Clin Periodontol. 1996;23(5):492-6. doi: 10.1111/j.1600-051X.1996.tb00580.x. [PubMed: 8783057].

35. Gur A, Sarac AJ, Cevik R, Altindag O, Sarac S. Efficacy of 904 nm gallium arsenide low level laser therapy in the management of chronic myofascial pain in the neck: a double-blind and randomize-controlled 
trial. Lasers Surg Med. 2004;35(3):229-35. doi: 10.1002/lsm.20082. [PubMed: 15389743].

36. Baltzer AW, Ostapczuk MS, Stosch D. Positive effects of low level laser therapy (LLLT) on Bouchard's and Heberden's osteoarthritis. Lasers Surg Med. 2016;48(5):498-504. doi: 10.1002/lsm.22480. [PubMed: 26833862].

37. Karu TI. Multiple roles of cytochrome c oxidase in mammalian cells under action of red and IR-A radiation. IUBMB Life. 2010;62(8):607-10. doi: 10.1002/iub.359. [PubMed: 20681024].

38. Bjordal JM, Johnson MI, Iversen V, Aimbire F, Lopes-Martins RA. Low-level laser therapy in acute pain: a systematic review of possible mechanisms of action and clinical effects in randomized placebo-controlled trials. Photomed LaserSurg. 2006;24(2):158-68. doi: 10.1089/pho.2006.24.158. [PubMed: 16706694].

39. Almeida-Lopes L, Rigau J, Zangaro RA, Guidugli-Neto J, Jaeger MM. Comparison of the low level laser therapy effects on cultured human gingival fibroblasts proliferation using different irradiance and same fluence. Lasers Surg Med. 2001;29(2):179-84. doi: 10.1002/lsm.1107. [PubMed: 11553908].

40. Bjordal JM, Lopes-Martins RA, Iversen VV. A randomised, placebo controlled trial of low level laser therapy for activated Achilles tendinitis with microdialysis measurement of peritendinous prostaglandin E2 concentrations. Br J Sports Med. 2006;40(1):76-80. doi: 10.1136/bjsm.2005.020842. [PubMed: 16371497] discussion 76-80. 